\section{Dementia

\title{
Lower Cognitive Function in Older Patients with Lower Muscle Strength and Muscle Mass
}

\author{
Romee van Dam ${ }^{a} \quad$ Jeanine M. Van Ancum ${ }^{a}$ Sjors Verlaan ${ }^{b}$ \\ Kira Scheerman $^{b}$ Carel G.M. Meskers ${ }^{c}$ Andrea B. Maier ${ }^{a, d}$ \\ a Department of Human Movement Sciences, @AgeAmsterdam, Vrije Universiteit, \\ Amsterdam Movement Sciences, Amsterdam, The Netherlands; ${ }^{b}$ Department of Internal \\ Medicine, Section of Gerontology and Geriatrics, VU University Medical Center, Amsterdam \\ Movement Sciences, Amsterdam, The Netherlands; ' $D$ Department of Rehabilitation \\ Medicine, VU University Medical Center, Amsterdam Movement Sciences, Amsterdam, \\ The Netherlands; ${ }^{\mathrm{d}}$ Department of Medicine and Aged Care, @AgeMelbourne, Royal \\ Melbourne Hospital, University of Melbourne, Melbourne, VIC, Australia
}

\section{Keywords}

Aging and cognition · Cognitive functioning · Functional abilities · Muscle $\cdot$ Hospitalization

\begin{abstract}
Background: Low muscle strength and muscle mass are associated with adverse outcomes in older hospitalized patients. The aim of this study was to assess the association between cognitive functioning and muscle strength and muscle mass in hospitalized older patients. Methods: This prospective inception cohort included 378 patients aged 70 years or older. At admission patients were assessed for cognitive functioning by use of the Six-Item Cognitive Impairment Test (6-CIT). Muscle strength and muscle mass were assessed using handheld dynamometry and segmental multifrequency bioelectrical impedance analysis, within $48 \mathrm{~h}$ after admission and on day 7, or earlier on the day of discharge. Results: The data of 371 patients (mean age \pm standard deviation $80.1 \pm 6.4$ years, $49.3 \%$ female) were available for analyses. The median (interquartile range) 6 -CIT score was $4(0-8)$ points. At admission, lower cognitive functioning was associated with lower muscle strength, lower skeletal muscle mass (SMM), lower appendicular lean mass, and lower SMM index. Cognitive functioning was not associated with change in muscle strength and muscle mass during hospitalization. Conclusion: This study further strengthens evidence for an association between lower cognitive functioning and lower muscle strength and muscle mass, but without a further decline during hospitalization.
\end{abstract}

R. van Dam and J.M. Van Ancum contributed equally to this research. 
van Dam et al.: Cognition and Muscle in Older Patients

\section{Introduction}

Low muscle strength and muscle mass (sarcopenia) are associated with adverse outcomes in older people, e.g., falls [1], functional impairments [2, 3], higher risk of hospitalization [4], longer length of hospital stay [5], higher readmission rate [5], and higher mortality [6]. Sarcopenia is present in $10-25 \%$ of older hospitalized patients [5, 7], but relies on the applied definition [8]. A significant decrease in muscle strength and muscle mass was found during hospitalization of electively admitted older patients [9].

Low cognitive functioning might be a predisposing factor for low muscle strength and muscle mass [10]. In community-dwelling older people, cross-sectional studies found a positive association between cognitive functioning and sarcopenia [11]. In longitudinally followed community-dwelling older people, low cognitive functioning was found to be associated with a faster decrease in hand grip strength (HGS) [12], and low HGS was found to be associated with a more rapid decrease in cognitive functioning [13] and a higher risk of developing Alzheimer disease [14]. A recent cross-sectional study showed an association between low cognitive functioning and low muscle strength and muscle mass in hospitalized older patients [15]. Whether cognitive functioning is associated with changes in muscle strength and muscle mass during hospitalization in older patients is unknown.

The aim of this study was to assess the association between cognitive functioning and muscle strength and muscle mass at admission and the change of muscle strength and muscle mass during hospitalization in older patients.

\section{Methods}

Study Design

The Evaluation of Muscle Parameters in a Prospective Cohort of Older Patients at Clinical Wards Exploring Relations with Bed Rest and Malnutrition (EMPOWER) is an observational, prospective, and longitudinal inception cohort study conducted from April to December 2015 at the VU University Medical Center in Amsterdam, The Netherlands. Patients were excluded if their expected length of stay was $<24 \mathrm{~h}$, if they were admitted to intensive or medium care, if they were nursed in an air pressure isolation room, if they did not understand the Dutch language, or if they were not able to give informed consent due to advanced dementia or severe delirium. In this study, 378 patients were included. A flowchart of patient inclusion is shown in Figure 1.

\section{Data Collection}

Patients were assessed at two occasions during hospitalization. The first assessment was performed within $48 \mathrm{~h}$ of admission and included recording of general patient characteristics and measurement of muscle strength and muscle mass. The second assessment was performed on the day of discharge or at the latest on day 7 after the first assessment and included re-measurement of muscle strength and muscle mass.

\section{Patient Characteristics}

Age, sex, number of comorbidities, and number of medications and attending medical specialism were collected from medical records. Further determinants were collected with bedside interviews: smoking habits, living situation, falls in the previous 6 months, risk of delirium assessed by asking for presence of memory problems, depending on care in the $24 \mathrm{~h}$ prior to admission, delirium during previous hospitalization, functional status measured by the Katz Index of Independence in Activities of Daily Living [16], height in cm estimated using knee height [17], and weight in $\mathrm{kg}$.

\section{Cognition}

Cognition was tested at admission using the Six-Item Cognitive Impairment Test (6-CIT) [18]. The 6-CIT is a short questionnaire and consists of six questions, one memory question (remembering an address), two calculation questions (recalling numbers and months backward), and three orientation questions (naming 


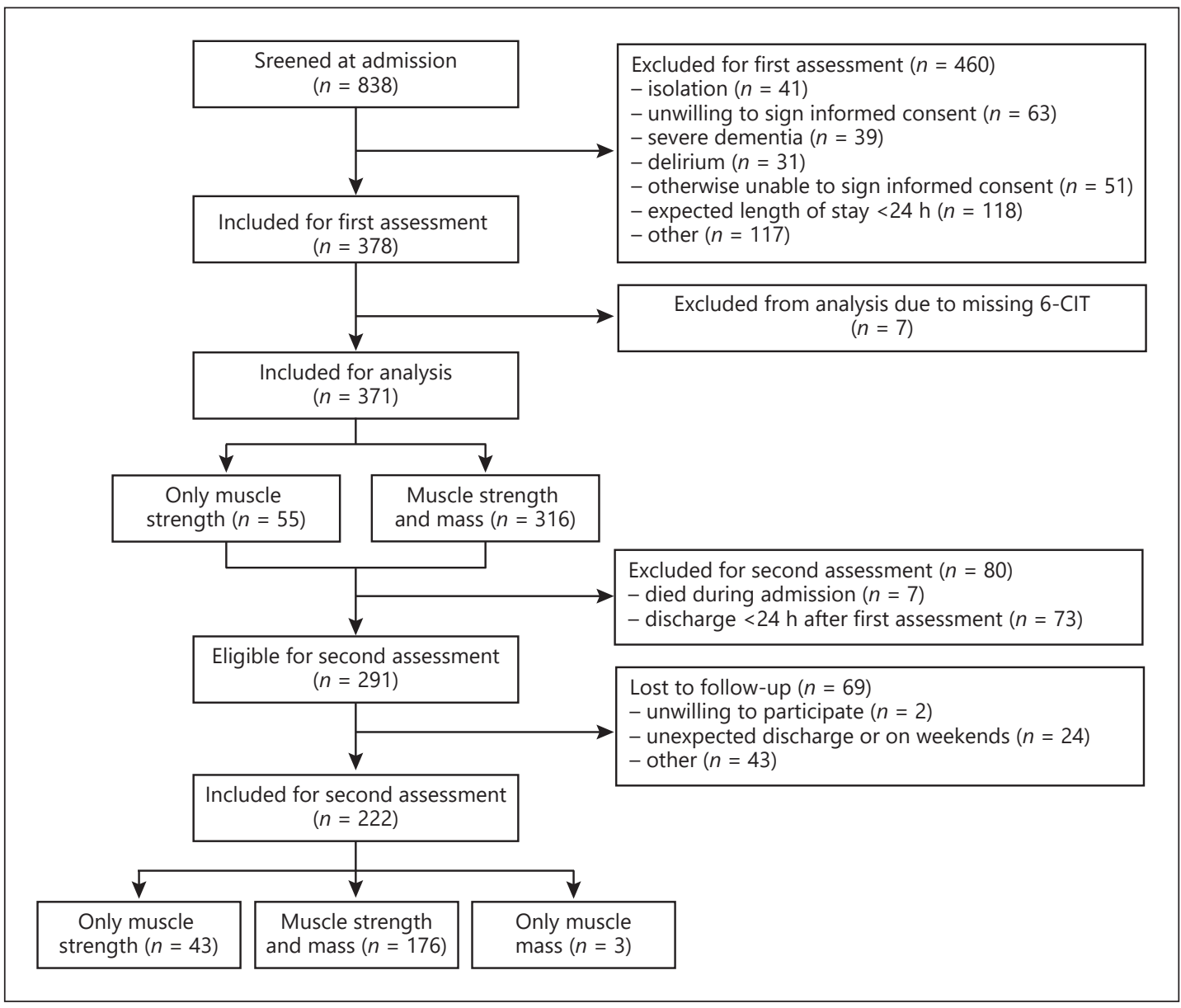

Fig. 1. Flowchart of patient inclusion. 6-CIT, Six-Item Cognitive Impairment Test.

of the time, the month, and the year). The 6-CIT is a validated questionnaire in an older emergency department population [18] and is based on the 26-item Blessed Information-Memory-Concentration Scale [19]. The score on the 6-CIT ranges from 0 to 28 and has been used as a continuous and dichotomous measure with a proposed cutoff value of $\geq 10$ points indicating low cognitive functioning [20].

\section{Muscle Parameters}

Muscle Strength. Muscle strength was estimated by HGS measured using a hydraulic handheld dynamometer (Jamar, Sammons Preston Rolyan, Bolingbrook, IL, USA). Patients were asked to sit upright with the elbows unsupported and in flexed position $\left(90^{\circ}\right)$. If patients were unable to sit, HGS was measured with the bed in a $30^{\circ}$ angle and with unsupported elbows. Each patient had two attempts on each side. Patients were asked to squeeze maximally and were encouraged by the assessor. The maximum score was used for further analysis.

Muscle Mass. Measurements of muscle mass were performed using direct segmental multifrequency bioelectrical impedance analysis (DSM-BIA, InBody S10, Biospace Co., Ltd., Seoul, Korea). DSM-BIA is a validated method for estimating skeletal muscle mass (SMM) compared to dual-energy X-ray absorptiometry [21]. Muscle mass was expressed as SMM in kg, appendicular lean mass (ALM) in kg, SMM index (SMI; SMM/ height ${ }^{2}$ in $\mathrm{kg} / \mathrm{m}^{2}$ ), relative SMM (SMM/weight) in percent, and relative ALM (ALM/weight) in percent.

\section{Statistical Analysis}

Variables with a normal distribution were presented as mean with standard deviation. Variables with a skewed distribution were presented as median with interquartile range. Categorical variables were 
Table 1. Characteristics of the study population $(n=371)$

Age, years

Female

Living independently

$>1$ comorbidities

$>4$ medications

Current smoking

Weight, kg

Height, $\mathrm{cm}^{\mathrm{a}}$

Risk of delirium ${ }^{\mathrm{b}}$

Falls in the last 6 months

Katz Index of Independence in Activities of Daily Living score $>1^{c}$

Six-Item Cognitive Impairment Test score

Elective admission

Nonsurgical admission

Onset of delirium during hospitalization ${ }^{\mathrm{d}}$

Length of stay, days

Time between measurements, days ${ }^{\mathrm{d}}$

Hand grip strength, kg

Skeletal muscle mass, kg

Appendicular lean mass, kg

Skeletal muscle mass index, $\mathrm{kg} / \mathrm{m}^{2}$

Relative skeletal muscle mass, $\%$

Relative appendicular lean mass, $\%$

$$
\begin{gathered}
80.1(6.4) \\
183(49.3 \%) \\
333(89.8 \%) \\
327(88.1 \%) \\
229(61.7 \%) \\
39(10.5 \%) \\
73.2(17.1) \\
168(9.5) \\
221(59.6 \%) \\
169(45.6 \%) \\
150(40.4 \%) \\
4(0-8) \\
57(15.4 \%) \\
212(57.1 \%) \\
32(8.6 \%) \\
5.0(3.0-8.0) \\
5.0(3.0-7.0) \\
20.6(9.9) \\
26.1(6.0) \\
20.1(5.5) \\
9.2(1.5) \\
36.4(6.0) \\
27.9(5.8)
\end{gathered}
$$

Variables are presented as mean (standard deviation), $n$ (\%), or median (interquartile range). ${ }^{\text {a }}$ Based on knee height and LASA formula. ${ }^{b}$ Risk of delirium in case one or more questions were answered positive: presence of memory problems, depending on care in the $24 \mathrm{~h}$ prior to admission, delirium during previous hospitalization. ${ }^{\mathrm{c}}$ Range $0-6,0=$ independent. ${ }^{\mathrm{d}}$ Assessed in 222 patients.

presented as number with percentage. Seven patients were excluded from the analysis due to missing 6-CIT score, leaving 371 patients for analysis (Fig. 1).

Linear regression analysis was used to test the associations between cognitive functioning and muscle strength and muscle mass at admission, and the change in muscle strength and muscle mass during hospitalization. Adjustments included sex, age (model 1), number of comorbidities, HGS for height, and relative muscle mass values for weight (model 2). The analysis of change in muscle strength and mass additionally included time between measurements, incidence of delirium during hospitalization, and HGS and muscle mass parameters at admission in model 2. Data were presented as beta with $95 \%$ confidence interval. The Statistical Package for the Social Sciences (IBM SPSS Statistics for Windows, version 23.0, IBM Corp., Armonk, NY, USA) was used for statistical analysis. $p$ values $<0.05$ were considered statistically significant.

\section{Results}

Table 1 shows the characteristics of the study population. The mean age was 80.1 years (standard deviation 6.4). The percentage of female patients was $49.3 \%$. The median (interquartile range) 6-CIT score was 4 (0-8) points and the median (interquartile range) length of stay was 5 (3-8) days. Similar results for baseline characteristics were found for both female and male patients.

Figure 2 shows the results of the associations between cognitive functioning and HGS and muscle mass at admission. Lower cognitive functioning was significantly associated with lower HGS, SMM, ALM, and SMI. No statistically significant associations were found between cognitive functioning and relative ALM and relative SMM. The detailed effect sizes of the 
van Dam et al.: Cognition and Muscle in Older Patients

Table 2. Association between cognitive functioning and change in HGS and muscle mass of patients during hospitalization $(n=$ 222)

\begin{tabular}{|c|c|c|c|c|c|c|}
\hline 6-CIT score & $\Delta \mathrm{HGS}, \mathrm{kg}$ & $\Delta \mathrm{SMM}^{\mathrm{a}}, \mathrm{kg}$ & $\Delta \mathrm{ALM}^{\mathrm{a}}, \mathrm{kg}$ & $\Delta \mathrm{SMI}^{\mathrm{a}}, \mathrm{kg} / \mathrm{m}^{2}$ & $\Delta$ Relative $\mathrm{SMM}^{\mathrm{a}}, \%$ & $\Delta$ Relative $\mathrm{ALM}^{\mathrm{a}}, \%$ \\
\hline $\begin{array}{l}\text { Model 1, B } \\
\quad(95 \% \mathrm{CI})\end{array}$ & $\begin{array}{l}0.04 \\
(-0.05 \text { to } 0.13)\end{array}$ & $\begin{array}{l}0.04 \\
(0.00 \text { to } 0.09)\end{array}$ & $\begin{array}{l}0.02 \\
(-0.02 \text { to } 0.07)\end{array}$ & $\begin{array}{l}0.02 \\
(0.00 \text { to } 0.03)\end{array}$ & $\begin{array}{l}0.07 \\
(0.01 \text { to } 0.14)\end{array}$ & $\begin{array}{l}0.05 \\
(-0.02 \text { to } 0.12)\end{array}$ \\
\hline $\begin{array}{c}\text { Model 2, B } \\
\quad(95 \% \mathrm{CI})\end{array}$ & $\begin{array}{l}-0.02 \\
(-0.11 \text { to } 0.08)\end{array}$ & $\begin{array}{l}0.04 \\
(-0.02 \text { to } 0.09)\end{array}$ & $\begin{array}{l}-0.01 \\
(-0.05 \text { to } 0.05)\end{array}$ & $\begin{array}{l}0.01 \\
(-0.01 \text { to } 0.03)\end{array}$ & $\begin{array}{l}0.05 \\
(-0.02 \text { to } 0.13)\end{array}$ & $\begin{array}{l}0.02 \\
(-0.06 \text { to } 0.10)\end{array}$ \\
\hline
\end{tabular}

Model 1: adjusted for sex and age. Model 2: Model 1 + adjusted for number of comorbidities, time between measurements, onset of delirium during hospitalization, HGS and muscle mass parameters at admission, HGS for height, and relative measures (relative SMM, relative ALM) for weight. Bold indicates statistical significance $(p<0.05)$. 6-CIT, Six-Item Cognitive Impairment Test; ALM, appendicular lean mass; CI, confidence interval; HGS, hand grip strength; SMI, skeletal muscle mass index; SMM, skeletal muscle mass. ${ }^{a}$ For measures of muscle mass: $n=179$.

Fig. 2. Association between cognitive functioning and HGS and muscle mass of patients at admission $(n=371)$. Bars depict predicted means, error bars 1 standard error. Adjusted for sex, age, number of comorbidities, HGS for height, and relative muscle mass value for weight. $* p<0.05, * * p<$ $0.01,{ }^{* * *} p<0.001 .6$-CIT, Six-Item Cognitive Impairment Test; ALM, appendicular lean mass; HGS, hand grip strength; SMI, skeletal muscle mass index; SMM, skeletal muscle mass.
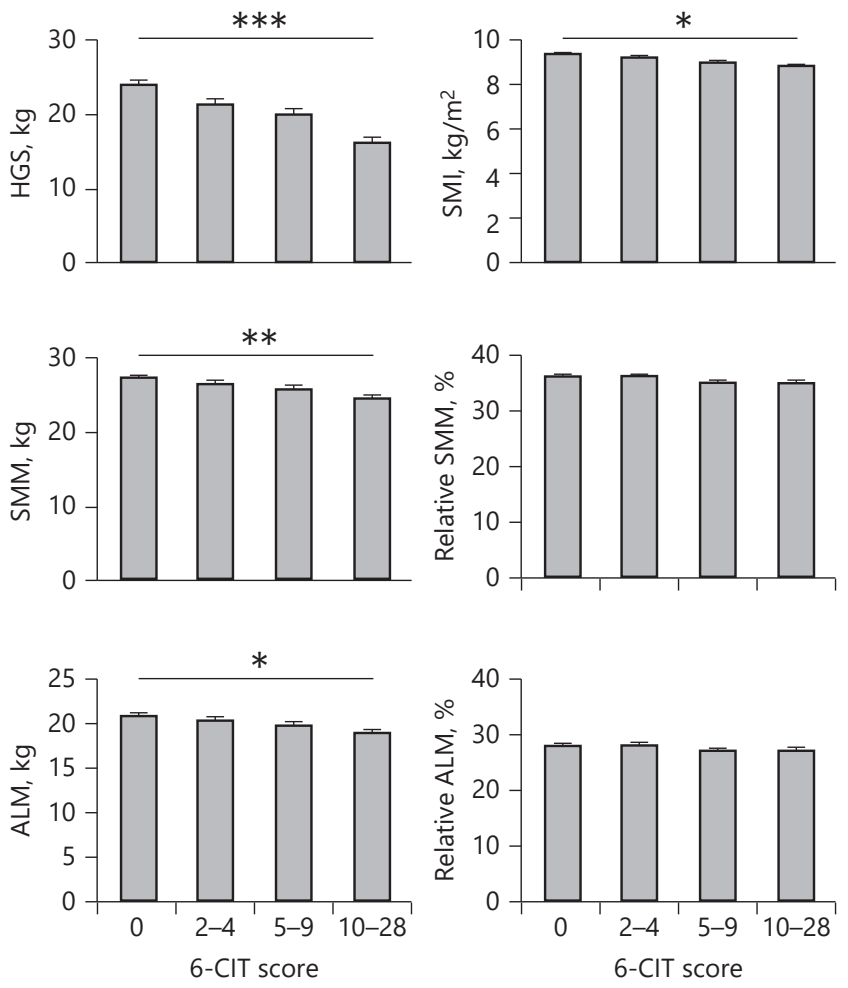

cross-sectional analyses can be found in online supplementary Table S1 (for all online suppl. material, see www.karger.com/doi/10.1159/000486711).

Descriptive statics of HGS and muscle mass at admission and discharge can be found in online supplementary Table S2. The associations between cognitive functioning and change in HGS and muscle mass during hospitalization are given in Table 2. No statistically significant association was found between cognitive functioning and change in HGS. Lower cognitive functioning was associated with a greater change towards a larger relative SMM after adjustment for sex and age. After further adjustment (model 2), this significant association was lost. 
van Dam et al.: Cognition and Muscle in Older Patients

\section{Discussion}

In this observational, prospective inception cohort study, lower cognitive functioning measured by the 6-CIT was associated with lower HGS, lower SMM, lower ALM, and lower SMI in older hospitalized patients at admission. Cognitive functioning was not associated with changes in muscle strength and muscle mass during hospitalization.

Cognitive impairment has been associated with less physical activity $[22,23]$ and inadequate dietary intake [24], leading to loss of muscle mass in affected people. Alternatively, several other common underlying causes for cognitive functioning and muscle strength and muscle mass have been reported, e.g., inflammation [25, 26], oxidative stress [27, 28], sex steroid levels [29, 30], and myokines [31]. Therefore, our finding that lower cognitive functioning is associated with lower HGS at admission is in line with the theoretical framework and with a recent cross-sectional study conducted in Japan that showed an association between low cognitive functioning and the presence of the combination of low HGS and low SMI in hospitalized older patients [15]. In this study, no data was presented for muscle strength and muscle mass separately. Two previous cross-sectional studies in communitydwelling older people showed an association between lower cognitive functioning and lower HGS $[12,13]$. We found that patients with lower cognitive functioning had a lower SMM, ALM, and SMI. In community-dwelling adults, an association was found between cognitive functioning and muscle mass in men [32], but not in women [33].

No associations were found between cognitive functioning and relative muscle mass. Differences between absolute and relative muscle measures can be explained by a confounding effect of body mass on the ratio between absolute and relative muscle mass, i.e., relative muscle mass tends to get lower in people with a higher total body mass [34]. In our population, patients with lower cognitive functioning had a lower total body mass, inducing a higher relative muscle mass. After adjustment for weight, the association between cognitive functioning and lower relative muscle measures got stronger.

In community-dwelling people, cognitive functioning was found to be associated with a faster decrease in HGS [12]. We expected that patients with lower cognitive functioning would be at higher risk for a decrease in muscle strength during hospitalization. However, this hypothesis could not be substantiated, neither for HGS nor for measures of muscle mass. Discrepancies may be explained by a short length of stay, with a subsequent short observation period. Long-term follow-up measurements after hospital stay are needed for further assessment of the relationship between cognition and muscle measures in populations of hospitalized patients and to address the effect of hospitalization itself.

\section{Strengths and Limitations}

To the best of our knowledge, the present study was the first to assess the relationship between cognitive functioning and change in muscle strength and muscle mass during hospitalization of older patients. Selection bias was minimized by the design of a clinically relevant inception cohort, applying a limited number of exclusion criteria. The average length of stay was short, and long-term follow-up for muscle mass and muscle strength was not available. We used the 6-CIT questionnaire as a proxy for cognitive functioning. The 6-CIT was recently validated [18], and this short questionnaire was easy to apply in the clinical setting. The use of DSM-BIA to measure muscle mass was easy but may have been influenced by the patients' hydration status. Since patients with advanced dementia were excluded and the median 6-CIT score of 4 (interquartile range $0-8$ ) in the present study population implies normal cognitive function [18], the impact of dementia on muscle measures was not examined in this study. We were not able to control for influential factors such as nutritional intake, physical activity, and treatments during hospitalization, which may have influenced our outcome measures. 
van Dam et al.: Cognition and Muscle in Older Patients

\section{Conclusion}

This observational, prospective inception cohort study in older hospitalized patients further strengthens evidence for an association between lower cognitive functioning as measured by the 6-CIT and lower HGS, lower SMM, lower ALM, and lower SMI at admission. No associations were found between cognitive functioning at admission and change in muscle strength and muscle mass during hospitalization. Further research with a longer follow-up period is recommended to re-assess the effects of hospitalization on muscle strength and muscle mass in older patients in relation to cognitive functioning.

\section{Statement of Ethics}

This study was approved by the Medical Ethics Committee of the VU University Medical Center and all included patients signed informed consent.

\section{Disclosure Statement}

The authors declare that they have no conflicts of interest.

\section{Funding Sources}

This work was supported by the seventh framework program MYOAGE [HEALTH-2007-2.4.5-10]; the European Union's Horizon 2020 research and innovation program [689238, 675003], and Nutricia Research, Nutricia Advanced Medical Nutrition, The Netherlands.

\section{Author Contributions}

Study concept and design: J.M. Van Ancum, K. Scheerman, C.G.M. Meskers, and A.B. Maier. Acquisition of data: R. van Dam, J.M. Van Ancum, K. Scheerman, C.G.M. Meskers, and A.B. Maier. Analysis and interpretation of data: R. van Dam, J.M. Van Ancum, K. Scheerman, S. Verlaan, C.G.M. Meskers, and A.B. Maier. Drafting of the manuscript: R. van Dam, J.M. Van Ancum, C.G.M. Meskers, and A.B. Maier. Critical revision of the manuscript for important intellectual content: R. van Dam, J.M. Van Ancum, K. Scheerman, S. Verlaan, C.G.M. Meskers, and A.B. Maier. Final approval of the version to be published: R. van Dam, J.M. Van Ancum, K. Scheerman, S. Verlaan, C.G.M. Meskers, and A.B. Maier. Study supervision: C.G.M. Meskers and A.B. Maier.

\section{References}

1 Szulc P, Beck TJ, Marchand F, Delmas PD: Low skeletal muscle mass is associated with poor structural parameters of bone and impaired balance in elderly men - the MINOS study. J Bone Miner Res 2005;20:721-729.

-2 Janssen I, Heymsfield SB, Ross R: Low relative skeletal muscle mass (sarcopenia) in older persons is associated with functional impairment and physical disability. J Am Geriatr Soc 2002;50:889-896.

3 Amigues I, Schott AM, Amine M, Gelas-Dore B, Veerabudun K, Paillaud E, Beauchet O, Rolland Y, Canouï Poitrine F, Bonnefoy M: Low skeletal muscle mass and risk of functional decline in elderly community-dwelling women: the prospective EPIDOS study. J Am Med Dir Assoc 2013;14:352-357.

4 Legrand D, Vaes B, Matheï C, Adriaensen W, Van Pottelbergh G, Degryse JM: Muscle strength and physical performance as predictors of mortality, hospitalization, and disability in the oldest old. J Am Geriatr Soc 2014; 62:1030-1038.

5 Gariballa S, Alessa A: Sarcopenia: prevalence and prognostic significance in hospitalized patients. Clin Nutr 2013;32:772-776.

6 Volaklis KA, Halle M, Meisinger C: Muscular strength as a strong predictor of mortality: a narrative review. Eur J Intern Med 2015;26:303-310.

7 Smoliner C, Sieber CC, Wirth R: Prevalence of sarcopenia in geriatric hospitalized patients. J Am Med Dir Assoc 2014;15:267-272. 
8 Reijnierse EM, Trappenburg MC, Leter MJ, Blauw GJ, Sipila S, Sillanpaa E, Narici MV, Hogrel JY, Butler-Browne G, McPhee JS, Gapeyeva H, Paasuke M, de van der Schueren MA, Meskers CG, Maier AB: The impact of different diagnostic criteria on the prevalence of sarcopenia in healthy elderly participants and geriatric outpatients. Gerontology 2015;61:491-496.

-9 Van Ancum JM, Scheerman K, Jonkman NH, Smeenk HE, Kruizinga RC, Meskers CGM, Maier AB: Change in muscle strength and muscle mass in older hospitalized patients: a systematic review and meta-analysis. Exp Gerontol 2017;92:34-41.

10 Christensen H, Mackinnon AJ, Korten A, Jorm AF: The "common cause hypothesis" of cognitive aging: evidence for not only a common factor but also specific associations of age with vision and grip strength in a crosssectional analysis. Psychol Aging 2001;16:588-599.

-11 Chang KV, Hsu TH, Wu WT, Huang KC, Han DS: Association between sarcopenia and cognitive impairment: a systematic review and meta-analysis. J Am Med Dir Assoc 2016;17:1164.e7-e15.

-12 Raji MA, Kuo YF, Snih SA, Markides KS, Peek MK, Ottenbacher KJ: Cognitive status, muscle strength, and subsequent disability in older Mexican Americans. J Am Geriatr Soc 2005;53:1462-1468.

-13 Alfaro-Acha A, Al Snih S, Raji MA, Kuo YF, Markides KS, Ottenbacher KJ: Handgrip strength and cognitive decline in older Mexican Americans. J Gerontol A Biol Sci Med Sci 2006;61:859-865.

14 Boyle PA, Buchman AS, Wilson RS, Leurgans SE, Bennett DA: Association of muscle strength with the risk of Alzheimer's disease and the rate of cognitive decline in community-dwelling older persons. Arch Neurol 2009; 66:1339-1344.

15 Maeda K, Akagi J: Cognitive impairment is independently associated with definitive and possible sarcopenia in hospitalized older adults: the prevalence and impact of comorbidities. Geriatr Gerontol Int 2017;17:10481056.

16 Reijneveld SA, Spijker J, Dijkshoorn H: Katz' ADL index assessed functional performance of Turkish, Moroccan, and Dutch elderly. J Clin Epidemiol 2007;60:382-388.

17 Han TS, Lean ME: Lower leg length as an index of stature in adults. Int J Obes Relat Metab Disord 1996;20: 21-27.

18 O’Sullivan D, Brady N, Manning E, O’Shea E, O’Grady S, O’Regan N, Timmons S: Validation of the 6-Item Cognitive Impairment Test and the 4AT test for combined delirium and dementia screening in older emergency department attendees. Age Ageing 2018;47:61-68.

19 Blessed G, Tomlinson BE, Roth M: The association between quantitative measures of dementia and of senile change in the cerebral grey matter of elderly subjects. Br J Psychiatry 1968;114:797-811.

-20 Upadhyaya AK, Rajagopal M, Gale TM: The Six Item Cognitive Impairment Test (6-CIT) as a screening test for dementia: comparison with Mini-Mental State Examination (MMSE). Curr Aging Sci 2010;3:138-142.

21 Bosaeus I, Wilcox G, Rothenberg E, Strauss BJ: Skeletal muscle mass in hospitalized elderly patients: comparison of measurements by single-frequency BIA and DXA. Clin Nutr 2014;33:426-431.

-22 Laurin D, Verreault R, Lindsay J, MacPherson K, Rockwood K: Physical activity and risk of cognitive impairment and dementia in elderly persons. Arch Neurol 2001;58:498-504.

23 Guimarães FC, Amorim PR, Reis FF, Bonoto RT, Oliveira WC, Moura TA, Assis CL, Palotás A, Lima LM: Physical activity and better medication compliance improve Mini-Mental State Examination scores in the elderly. Dement Geriatr Cogn Disord 2015;39:25-31.

24 Wirth R, Smoliner C, Sieber CC, Volkert D: Cognitive function is associated with body composition and nutritional risk of geriatric patients. J Nutr Health Aging 2011;15:706-710.

25 Visser M, Pahor M, Taaffe DR, Goodpaster BH, Simonsick EM, Newman AB, Nevitt M, Harris TB: Relationship of interleukin- 6 and tumor necrosis factor-alpha with muscle mass and muscle strength in elderly men and women: the Health ABC Study. J Gerontol A Biol Sci Med Sci 2002;57:M326-M332.

-26 Licastro F, Pedrini S, Caputo L, Annoni G, Davis LJ, Ferri C, Casadei V, Grimaldi LME: Increased plasma levels of interleukin-1, interleukin- 6 and $\alpha$-1-antichymotrypsin in patients with Alzheimer's disease: peripheral inflammation or signals from the brain? J Neuroimmunol 2000;103:97-102.

27 Meng SJ, Yu LJ: Oxidative stress, molecular inflammation and sarcopenia. Int J Mol Sci 2010;11:1509-1526.

28 Mariani E, Polidori MC, Cherubini A, Mecocci P: Oxidative stress in brain aging, neurodegenerative and vascular diseases: an overview. J Chromatogr B Analyt Technol Biomed Life Sci 2005;827:65-75.

29 Baumgartner RN, Waters DL, Gallagher D, Morley JE, Garry PJ: Predictors of skeletal muscle mass in elderly men and women. Mech Ageing Dev 1999;107:123-136.

-30 Hogervorst E, Bandelow S, Combrinck M, Smith AD: Low free testosterone is an independent risk factor for Alzheimer's disease. Exp Gerontol 2004;39:1633-1639.

31 Phillips C, Baktir MA, Srivatsan M, Salehi A: Neuroprotective effects of physical activity on the brain: a closer look at trophic factor signaling. Front Cell Neurosci 2014;8:170.

-32 Auyeung TW, Kwok T, Lee J, Leung PC, Leung J, Woo J: Functional decline in cognitive impairment - the relationship between physical and cognitive function. Neuroepidemiology 2008;31:167-173.

33 Abellan van Kan G, Cesari M, Gillette-Guyonnet S, Dupuy C, Nourhashémi F, Schott AM, Beauchet O, Annweiler C, Vellas B, Rolland Y: Sarcopenia and cognitive impairment in elderly women: results from the EPIDOS cohort. Age Ageing 2013;42:196-202.

-34 Bijlsma AY, Meskers MCG, Molendijk M, Westendorp RGJ, Sipilä S, Stenroth L, Sillanpää E, McPhee JS, Jones DA, Narici M, Gapeyeva H, Pääsuke M, Seppet E, Voit T, Barnouin Y, Hogrel JY, Butler-Browne G, Maier AB: Diagnostic measures for sarcopenia and bone mineral density. Osteoporos Int 2013;24:2681-2691. 